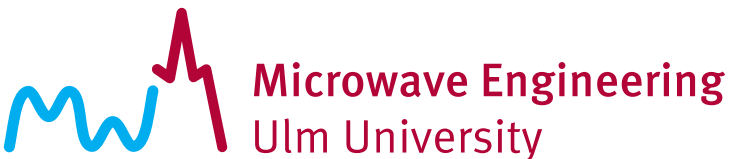

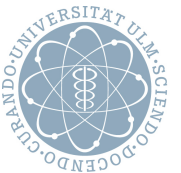

\section{On Hardware Implementations of Stepped-Carrier OFDM Radars}

\author{
Benedikt Schweizer, Daniel Schindler, Christina Knill, Jürgen Hasch, and Christian Waldschmidt
}

(C) 2018 IEEE. Personal use of this material is permitted. Permission from IEEE must be obtained for all other uses, in any current or future media, including reprinting/republishing this material for advertising or promotional purposes, creating new collective works, for resale or redistribution to servers or lists, or reuse of any copyrighted component of this work in other works.

DOI: 10.1109/MWSYM.2018.8439179 


\title{
On Hardware Implementations of Stepped-Carrier OFDM Radars
}

\author{
Benedikt Schweizer, Daniel Schindler, Christina Knill, Jürgen Hasch, and Christian Waldschmidt \\ B. Schweizer, C. Knill and C. Waldschmidt are with Ulm University, Ulm, Germany \\ D. Schindler and J. Hasch are with the Corporate Sector Research and Advance Engineering of \\ Robert Bosch GmbH, 70465 Stuttgart, Germany
}

\begin{abstract}
Orthogonal frequency-division multiplexing (OFDM) is a promising candidate for future digital-centric radar systems. One major limitation for practical implementations is the required sampling rate in the order of $\mathrm{GHz}$, which can be solved by using a stepped-carrier technique in combination with OFDM signals of smaller bandwidth, theoretically resulting in the same resolution as a conventional OFDM scheme. However, a signal source capable of generating such a carrier has to fulfill several constraints. In this paper, the effects of deterministic and random inaccuracies of the carrier generation as well as effects of phase noise are described and evaluated. A transient model that describes the switching between carriers is given and all effects are verified using a radar demonstrator at $77 \mathrm{GHz}$.
\end{abstract}

Index Terms - frequency-agility, OFDM, phase noise, PLL, radar, stepped carrier, transient behavior.

\section{INTRODUCTION}

Radar schemes based on linear frequency modulation have inherent limitations when high resolution and large unambiguous velocities are desired, as highly linear fast ramps of large bandwidth are hardly realizable, expecially in combination with time-division multiplexing (TDM) based multiple-input multiple-output (MIMO) configurations.

With the possibility to use interleaved FDM-MIMO, orthogonal frequency-division multiplexing (OFDM) based schemes offer a solution to overcome those limitations [1]. Furthermore, the requirements on the analog frontend are reduced as signal generation and evaluation are shifted to the digital domain. One current limitation that obstructs the utilization of OFDM in low-cost applications was stated to be the requirement to sample signals of the large channel bandwidth in baseband. A solution is called stepped-carrier OFDM, as presented in [2], where a method based on an agile carrier is proposed that generates the large RF bandwidth artificially in multiple steps. Such a method shifts the challenges of the hardware implementation to the carrier generation. This paper addresses those specific tasks and validates the requirements with simulations and measurements.

\section{STEPPED-CARRIER OFDM SCHEME}

An OFDM symbol consisting of $\mathcal{N}$ subcarriers that are coded with the QPSK modulation symbols $d_{n}$ and spaced by $\Delta f=1 / T$, can be described as

$$
x(t)=\sum_{n=0}^{\mathcal{N}-1} d_{n} \mathrm{e}^{\mathrm{j} 2 \pi n \Delta f t} \operatorname{rect}\left(\frac{t}{T}\right) .
$$

After generation via IDFT, a cyclic prefix of duration $T_{\mathrm{cp}}$ is added. $\mathcal{M}$ such symbols form the OFDM transmit signal,
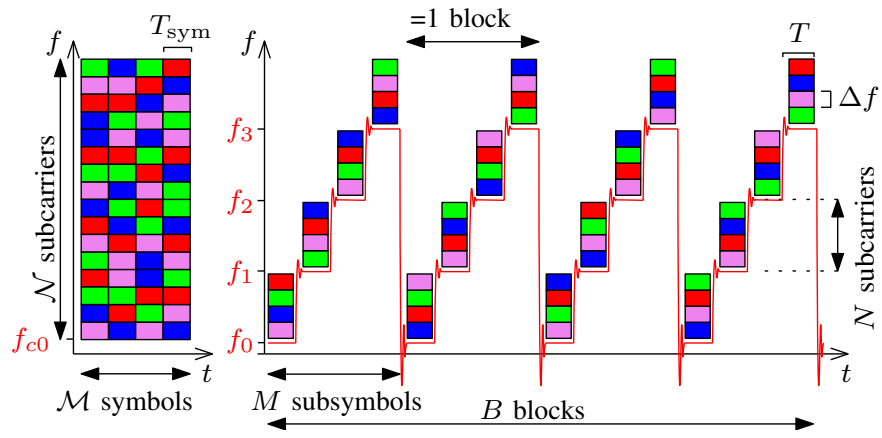

Fig. 1. Structure of OFDM signals in frequency domain, colors represent QPSK codes. Left: Standard OFDM. Right: Stepping-scheme in RF domain with parameters and a non-ideal realization of the stepped carrier (red). Adapted from [3].

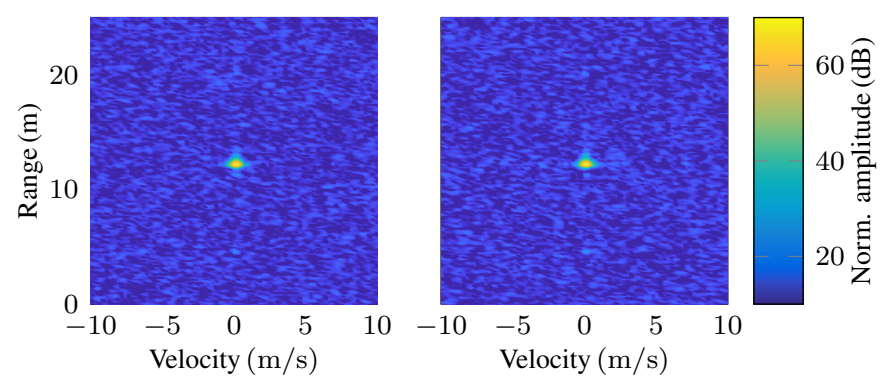
(a) 1 step
(b) 8 steps

Fig. 2. Measurement results for an ideal carrier with (a) $M=1$ step, (b) $M=8$ steps. The measurement setup and scene are according to Section III.

which is up-converted to the RF domain and transmitted. In the channel, the signal experiences the delay $\tau=(2 r-v t) / c_{0}$ for a single point scatterer at distance $r$ and its velocity $v$. After reception and conversion to baseband, the evaluation of such a signal is done in frequency domain. Therefore, the signal is sampled and each symbol is transformed to frequency domain. A spectral division by the transmitted phase codes yields a matrix $D$ with its elements

$$
D_{n m}=\mathrm{e}^{-\mathrm{j} 2 \pi \frac{2 R_{0}}{c_{0}}\left(f_{\mathrm{c} 0}+n \Delta f\right)} \mathrm{e}^{\mathrm{j} 2 \pi \frac{2 v m\left(T+T_{\mathrm{cp}}\right)}{c_{0}}\left(f_{\mathrm{c} 0}+n \Delta f\right)}
$$

that contain the desired channel information at the carrier frequency $f_{c 0}$. The target range and velocity is obtained with two (I)DFTs over symbols and subcarriers.

Instead of using $\mathcal{N}$ subcarriers and transmitting $\mathcal{M}$ symbols consecutively, the stepped-carrier approach uses $M$ subsym- 


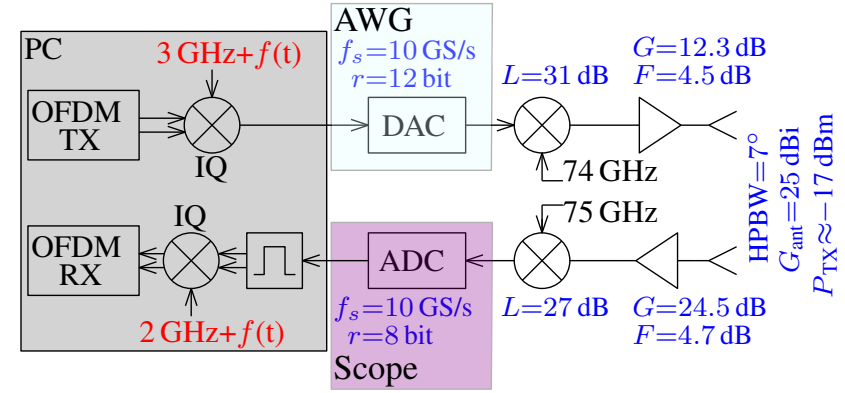

Fig. 3. Schematic of the heterodyne OFDM Radar demonstrator at $77 \mathrm{GHz}$. Adapted from [3]

bols of $N=\mathcal{N} / M$ subcarriers and changes the carrier frequency after each subsymbol according to

$$
f_{m}=f_{\mathrm{c} 0}+m N \Delta f,
$$

generating a large measurement bandwidth artificially. In [3], it is shown that the processing of a stepped-carrier OFDM scheme as shown in Fig. 1 can be done similarly by considering the actual position of the symbols in the timefrequency pattern. In that way, the range migration throughout one subsymbol is corrected inherently and a high-resolution range-velocity profile is obtained, as shown in Fig. 2. It is independent of the number of targets and does not increase the computational effort, only the unambiguous velocity is decreased by the factor of $M$ compared to a conventional scheme. For mathematical details, the reader is referred to [3].

\section{VERIFICATION AND MEASUREMENT CONCEPT}

To verify the scheme and the requirements on the stepped carrier, the effects are simulated in equivalent complex baseband domain and verified with an OFDM radar setup operating at $77 \mathrm{GHz}$ which is presented in [3]. It is a heterodyne setup with digital IF stage and image suppression based on different carrier frequencies for up- and down-conversion such that the upper and lower sideband do not overlap in baseband after analog down-conversion. The setup is depicted in Fig. 3. The signal generation is done with an arbitrary waveform generator (AWG), and for sampling a fast oscilloscope is used. This method allows to include the investigated carrier nonidealities in the digital IF stage, marked as $+f(t)$ in Fig. 3. All measurements use $M=8$ subsymbols consisting of $N=256$ subcarriers. Those are spaced by $\Delta f=500 \mathrm{kHz}$ and coded with QPSK modulation symbols. The duration of the cyclic prefix is $T_{\mathrm{cp}}=0.5 \mu \mathrm{s}$ and $B=384$ blocks are used resulting in an RF bandwidth of $B_{W}=1.024 \mathrm{GHz}$. The measurement involves a single target with an RCS of $\sigma_{\mathrm{RCS}}=17.5 \mathrm{dBsm}$ at $r=8 \mathrm{~m}$ which appears at $r=12.2 \mathrm{~m}$ due to the length of cables. This offset is not corrected since the actual time of flight is relevant for transient effects.

\section{INFLUENCE OF CARRIER PRECISION}

In a classic OFDM scheme, all subcarriers are generated digitally, resulting in a precise realization of $\Delta f$. By introducing a stepped carrier, the individual carrier frequencies

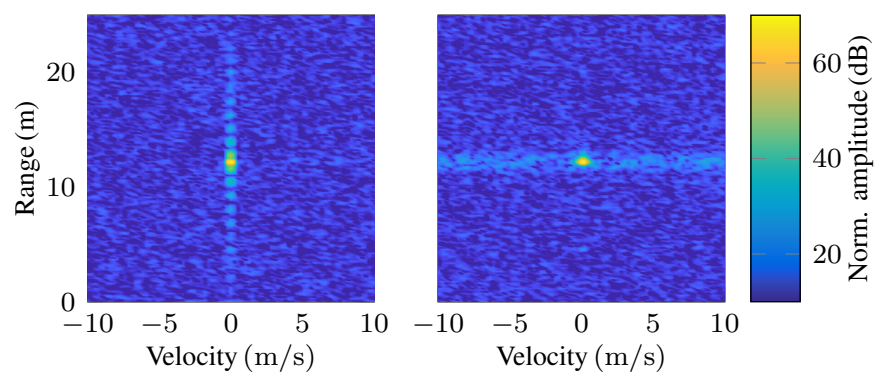

(a) Deterministic offset

(b) Random error

Fig. 4. Measurement results for the influence of deterministic offsets (a), and random errors (b) with $\sigma\left(\Delta f_{m, b}\right)=500 \mathrm{kHz}$.

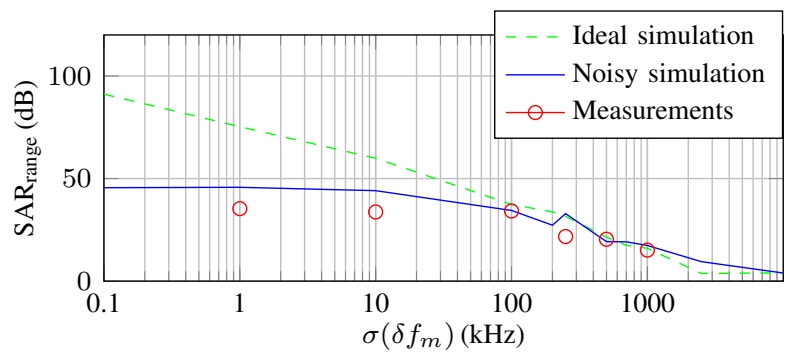

Fig. 5. Simulations and measurements for signal-to-artifact ratio (SAR) for deterministic offset depending on $\sigma=\delta f_{m}$ that is individual for each subsymbol $(m)$, but constant over time $(b)$.

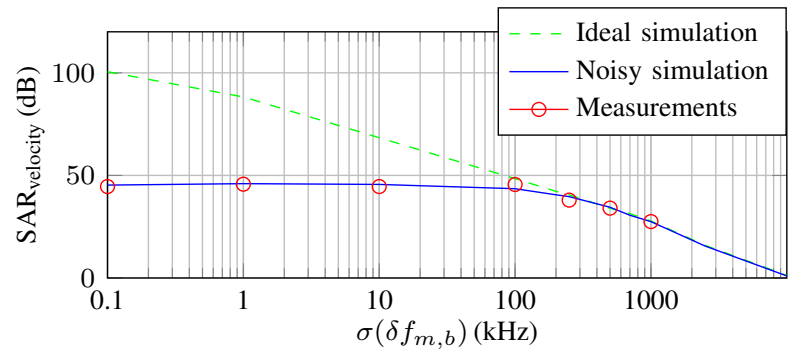

Fig. 6. Simulations and measurements for signal-to-artifact ratio (SAR) for deterministic offset depending on $\sigma=\delta f_{m, b}$ that is individual for each subsymbol $(m)$ and over time $(b)$.

need to be placed such that all virtual subcarriers are on a common frequency grid. If this condition is fulfilled, a radar image that equals a conventional OFDM scheme is obtained, as shown in Fig. 2. However, each carrier position $f_{m}$ can have a deterministic offset $\delta f_{m}$ that is constant over time, i.e., all blocks have the same error, leading to

$$
\tilde{f}_{m, b}=f_{m}+\delta f_{m} .
$$

Such an error may be caused by faulty discrete frequency positions of a tunable oscillator or inaccuracies in the reference oscillator of a PLL. This error is modeled with a Gaussian distribution, characterized by the standard deviation $\sigma$. Fig. 4a shows a measured range-velocity profile for $\sigma\left(\delta f_{m, b}\right)=500 \mathrm{kHz}$, which is equal to the subcarrier spacing. Such deterministic frequency offsets cause artifacts in range as the spacing of the virtual subcarriers is not constant anymore. 


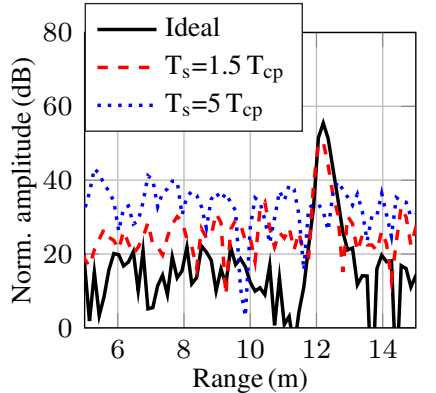

(a) Range profiles at $v=0$

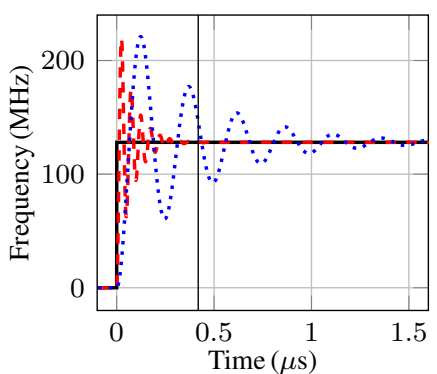

(b) Transient behaviors of carrier
Fig. 7. (a): Measured range profiles depending on normalized settling time of transients. (b): Transient course of the carrier frequency in baseband that is used for Measurements in (a). Vertical black line shows the maximum tolerable settling time for a target at $r=12.2 \mathrm{~m}$.

However, the Doppler evaluation is not affected as the offset is constant over time (independent of $b$ ).

Additionally, all realizations can be affected by a random error $\delta f_{m, b}$ that accounts for e.g. settling errors and alters all individual positions throughout time and frequency. The faulty carrier frequency

$$
\tilde{f}_{m, b}=f_{m}+\delta f_{m, b}
$$

results in a ridge in velocity direction, as shown in Fig. 4b. The range evaluation is barely affected since the calculation of the velocity profile averages the faulty carrier position to the mean value $f_{m}$.

The behavior is quantified in Fig. 5 where the signal-toartifact ratio (SAR) is depicted depending on $\sigma\left(f_{m}\right)$ for a deterministic offset and in Fig. 6 for a random error $\sigma\left(f_{m, b}\right)$. The measured points show good agreement with simulation results that take the same thermal noise level into account. The artifacts increase with roughly $20 \mathrm{~dB}$ per decade as soon as the thermal noise floor is not the limiting factor anymore. Simulations without additive Gaussian noise show that even small errors directly affect the achievable SAR. Thus, the desired dynamic range defines the tolerable offsets such that the artifacts are below the noise level.

\section{TRANSIENT BEHAVIOR}

In a conventional OFDM scheme, the requirements on the transient behavior of the carrier are neglectable as it only needs to settle to the desired value once at the beginning of operation. However, if a stepped carrier is used, switching the frequency requires the settling time $T_{\mathrm{s}}$ until the PLL has settled to the desired frequency. Due to the time of flight to a target, the portion of the OFDM signal that is transmitted during that period is up- and down-converted with two different instantaneous carrier frequencies. For the OFDM modulation, this results in a phase error that varies throughout the symbol duration, and for large deviations it deteriorates the orthogonality.

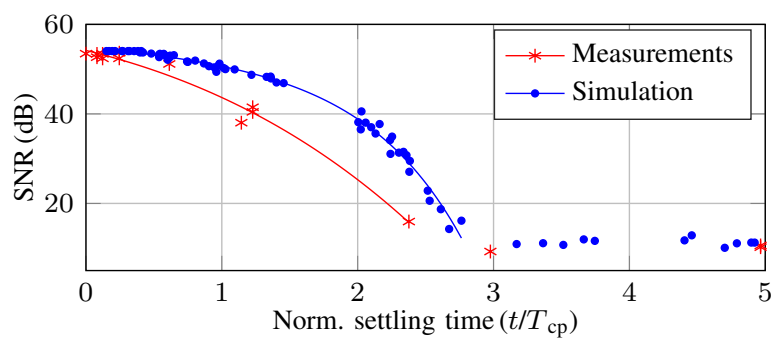

Fig. 8. Evaluation of dependency of target SNR on normalized settling time for measurements $(*)$ and simulations $(\bullet)$ with exponential fit.

To analyze the behavior independently of a specific PLL architecture, a $2^{\text {nd }}$ order low pass filter with the transfer function

$$
H(s)=\frac{\omega_{n}^{2}}{s^{2}+2 \xi \omega_{n} s+\omega_{n}^{2}}
$$

is used to model the non-ideal transients of the stepped carrier by filtering the ideal course of the stepped-frequency pattern in time domain. The parameters $\omega_{n}$ and $\xi$ allow to define the behavior in terms of settling time and overshoots. The resulting frequency pattern is used as IF carrier for measurements.

The results of those measurements are presented in Fig. 7a for three different settling behaviors that are shown in Fig. 7b. It is observable that a long settling time increases the noise level and reduces the target SNR. This relation is pointed out in Fig. 8, where the target SNR is plotted versus settling time, that is defined as $f_{\text {error }}\left(T_{\mathrm{s}}\right)=0.1 \Delta f=50 \mathrm{kHz}$. That duration is the maximum tolerable Doppler shift [1], [4]. Both measured and simulated results decrease exponentially with $T_{\mathrm{s}}$. The degradation is independent of the actual course of the transient and it is more prominent for larger distances as they occupy a longer portion of $T_{\mathrm{cp}}$.

As a consequence, an additional time that accounts for the settling time needs to be added to the cyclic prefix, what lowers the unambiguous measurable velocity. Thus, more advanced techniques for fast hopping are necessary to keep the settling time below a fraction of the symbol duration $T$.

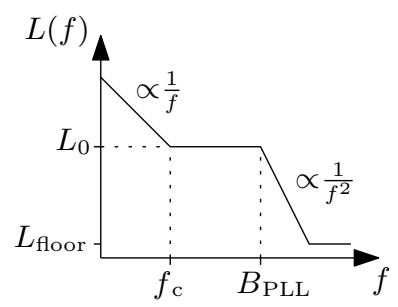

(a)

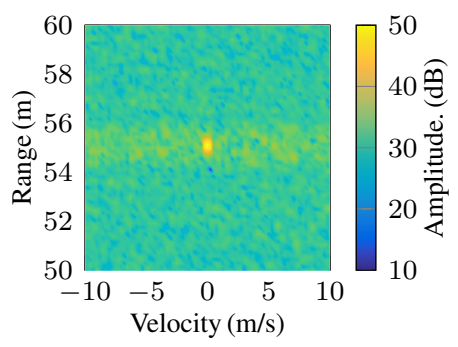

(b)
Fig. 9. (a): Generic PLL phase noise spectrum (b): Simulation result including phase noise for a single target at $r=55 \mathrm{~m}$. 


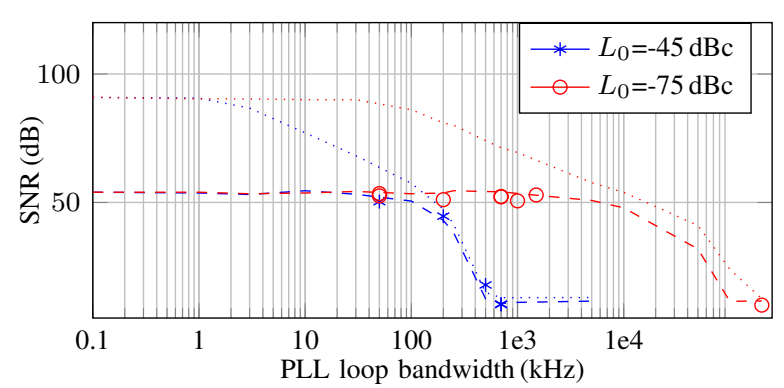

Fig. 10. Target SNR depending on $B_{P L L}$ for $L_{0}=-75 \mathrm{dBc} / \mathrm{Hz}$, $L_{\text {floor }}=-120 \mathrm{dBc} / \mathrm{Hz}, f_{c}=1 \mathrm{kHz}$. $(\circ, *)$ : Measurements $(\cdots)$ : Simulation without thermal noise. (- - ): Simulation with thermal noise.

\section{Phase NoISE}

As the requirements on the carrier are more challenging in a stepped-carrier OFDM scheme, the PLL cannot be optimized for lowest phase noise but has to meet the previously analyzed demands as well. Thereby, negative effects of phase noise become more relevant. The characteristic phase noise spectrum of a PLL, as shown in Fig. 9a, can be characterized by the $1 / f$ corner frequency $f_{c}$, in-band noise $L_{0}$, loop-bandwidth $B_{\mathrm{PLL}}$, and the out-of-band noise $L_{\text {floor }}$, according to

$$
L(f)=\frac{B_{\mathrm{PLL}}^{2} L_{0}}{B_{\mathrm{PLL}}^{2}+f^{2}}\left(1+\frac{f_{\mathrm{c}}}{f}\right)+L_{\text {floor }} .
$$

The time-domain phase jitter that corresponds to the phase noise spectrum is obtained by an IDFT of a realization of $L(f)$ with randomized phases. The phase jitter is added to the carriers of the IF stage of the heterodyne transceiver for measurements. This is possible as the phase noise of the measuring devices is much lower than the one under consideration. Phase noise causes two major effects. Firstly, a phase-noise floor is created, which can exceed the thermal noise level. This effect is caused by the variance of the carrier during one symbol. Secondly, a ridge in direction of velocity occurs, which becomes more prominent with increasing distance, exemplarily shown for the simulation of a target at $r=55 \mathrm{~m}$ in Fig. 9b. The second effect is caused by a decreasing similarity of the carrier realizations of different blocks for an increasing range. These phase errors correspond to arbitrary velocities.

Due to the phase noise floor, the observable SNR is limited by the strongest target. This behavior is evaluated in Fig. 10 depending on the loop bandwidth of the PLL and in Fig. 11 for different levels of the in-band noise $L_{0}$ and the out-ouf-band noise $L_{\text {floor }}$. Simulations without thermal noise show that phase noise limits the achievable SNR. However, the measurements and realistic simulations that include thermal noise are purely limited by thermal noise for reasonable parameterizations. As a consequence, the desired dynamic range defines the requirements on phase noise.

According to [5], $B_{\text {PLL }}$ should be smaller than $\Delta f / 2$ in order to avoid inter-subcarrier interference. The ideal simulation in Fig. 10 confirms this requirement, as the SNR increases degradation at approximately $100 \mathrm{kHz}$.

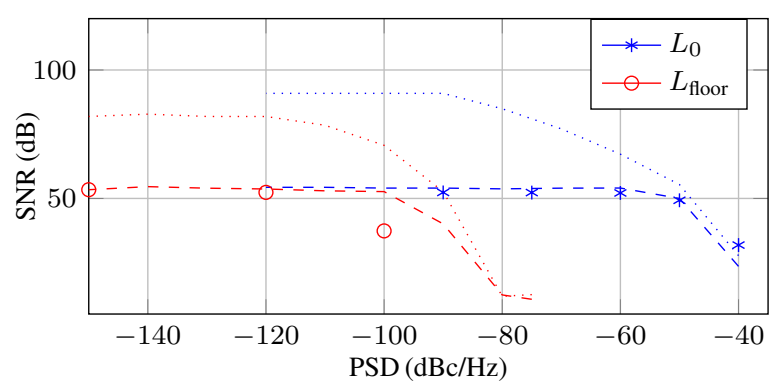

Fig. 11. SNR of a single target depending on $L_{0}$ and $L_{\text {floor }}$ for $L_{0}=-75 \mathrm{dBc} / \mathrm{Hz}, \quad L_{\text {floor }}=-120 \mathrm{dBc} / \mathrm{Hz}, \quad B_{\mathrm{PLL}}=200 \mathrm{kHz}, \quad f_{c}=1 \mathrm{kHz}$. $(\circ, *)$ : Measurements $(\cdots)$ : Simulation without thermal noise. (- - -): Simulation with thermal noise.

\section{CONCLUSION}

The stepped-carrier OFDM scheme offers the same range and velocity resolution as a conventional broadband OFDM scheme by use of affordable AD/DA-converters without increased computational effort. The challenges of a hardware implementation are shifted to the realization of the stepped carrier with fast settling time, accurate frequency positions and low phase noise. Thus, the implementation of such a scheme is only possible with detailed knowledge about all error sources. Switching transients require a defined guard time, as small violations of the cyclic prefix directly decrease the target SNR. Inaccurate frequency positions cause artifacts in range and velocity. As the theoretical limit is much above the achievable SNR due to thermal noise, an error below $100 \mathrm{kHz}$ is mostly tolerable. Phase noise creates a noise level that can exceed the thermal noise, and a ridge in velocity is observable, as it is the case for a conventional OFDM scheme.

\section{ACKNOWLEDGMENT}

The authors would like to thank the German Federal Ministry of Education and Research (BMBF), which partly funded the activities presented in this paper within the scope of the projects THINGS2DO (16ES0505) and autoSWIFT (16ES03).

\section{REFERENCES}

[1] C. Sturm, Y. L. Sit, M. Braun, and T. Zwick, "Spectrally interleaved multicarrier signals for radar network applications and multi-input multi-output radar," IET Radar, Sonar Navig., vol. 7, no. 3, pp. 261-269, Mar. 2013.

[2] G. Lellouch, A. K. Mishra, and M. Inggs, "Stepped OFDM radar technique to resolve range and Doppler simultaneously," IEEE Trans. Aerosp. Electron. Syst., vol. 51, no. 2, pp. 937-950, Apr. 2015.

[3] B. Schweizer, C. Knill, D. Schindler, and C. Waldschmidt, "Steppedcarrier OFDM-radar processing scheme to retrieve high-resolution rangevelocity profile at low sampling rate," IEEE Trans. Microw. Theory Tech., vol. 66, no. 3, pp. 1610-1618, Mar. 2018.

[4] G. Franken, H. Nikookar, and P. van Genderen, "Doppler tolerance of OFDM-coded radar signals," in Proc. 2006 Eur. Radar Conf., pp. 108111.

[5] A. G. Armada, "Understanding the effects of phase noise in orthogonal frequency division multiplexing (OFDM)," IEEE Transactions on Broadcasting, vol. 47, no. 2, pp. 153-159, Jun. 2001. 